Very high aspect ratio through-silicon vias (TSVs) fabricated using automated magnetic assembly of nickel wires

This article has been downloaded from IOPscience. Please scroll down to see the full text article.

2012 J. Micromech. Microeng. 22105001

(http://iopscience.iop.org/0960-1317/22/10/105001)

View the table of contents for this issue, or go to the journal homepage for more

Download details:

IP Address: 130.237.37.211

The article was downloaded on 22/08/2012 at 12:05

Please note that terms and conditions apply. 


\title{
Very high aspect ratio through-silicon vias (TSVs) fabricated using automated magnetic assembly of nickel wires
}

\author{
A C Fischer, S J Bleiker, T Haraldsson, N Roxhed, G Stemme \\ and F Niklaus
}

Microsystem Technology Laboratory, School of Electrical Engineering, KTH Royal Institute of

Technology, Osquldas väg 10, SE-100 44 Stockholm, Sweden

E-mail: andreas.fischer@ee.kth.se

Received 11 May 2012, in final form 28 June 2012

Published 17 August 2012

Online at stacks.iop.org/JMM/22/105001

\begin{abstract}
Through-silicon via (TSV) technology enables 3D-integrated devices with higher performance and lower cost as compared to 2D-integrated systems. This is mainly due to smaller dimensions of the package and shorter internal signal lengths with lower capacitive, resistive and inductive parasitics. This paper presents a novel low-cost fabrication technique for metal-filled TSVs with very high aspect ratios $(>20)$. Nickel wires are placed in via holes of a silicon wafer by an automated magnetic assembly process and are used as a conductive path of the TSV. This metal filling technique enables the reliable fabrication of through-wafer vias with very high aspect ratios and potentially eliminates characteristic cost drivers in the TSV production such as advanced metallization processes, wafer thinning and general issues associated with thin-wafer handling.
\end{abstract}

(Some figures may appear in colour only in the online journal)

\section{Introduction}

3D-integrated system in package (3D-SiP) solutions, which are based on vertical chip stacking, are a general trend in electronics and MEMS packaging. Not only do 3D-SiPs decrease device cost by reducing the volume and weight of the package, but they also improve the system performance through enhanced signal transmission speed and lower power consumption, which is important for various demanding applications [1, 2]. Different technologies for the electrical interconnection of stacked dies exist, such as wire bonding, flip-chip bonding and through-silicon vias (TSVs). In particular, TSVs enable shorter signal path lengths with superior electrical characteristics in terms of lower capacitive, resistive and inductive parasitic components [3]. Therefore, large development efforts for the realization of reliable and cost-efficient TSVs are currently ongoing and first commercially available devices such as MEMS inertial sensors and microphones, CMOS imagers and power LEDs successfully incorporate TSV technology [4-6].
The structure and hence the fabrication of TSVs can be roughly divided into three major elements: a vertical hole through the substrate, a conductive core and a dielectric layer acting as an insulator between the conductor and the substrate. The most common fabrication techniques of these elements are briefly discussed in the following subsections.

Via holes. Various methods for the formation of via holes exist and can be categorized into dry etching [1, 7-13], wet etching [8] and drilling processes [4, 14]. Via holes can have either straight $[9,10,12,13,15]$ or tapered sidewall profiles $[7,11]$ as well as combinations of both $[8,1]$. Typical diameters of via holes vary between a few microns [2,9] and several hundreds of microns $[12,15]$. The majority of TSVs have an aspect ratio between 1 and 10. Deep reactive ion etching (DRIE) is by far the most commonly used technology to form TSV holes. DRIE offers an excellent process controllability and is capable of creating high aspect ratio vias with specific sidewall profiles and topographies. The etch rate of DRIE is aspect ratio dependent and may cause several topographic imperfections on the sidewalls of the via holes such as scalloping, caused by alternating etch and passivation steps, 
Table 1. Relative permittivity $\epsilon_{r}$ and mechanical properties

(Young's modulus $E$ and coefficient of thermal expansion (CTE)) of commonly used TSV insulation materials, including silicon nitride and silicon oxide as well as emerging low-k insulators. Silicon serves as reference in the first row.

\begin{tabular}{llcl}
\hline Material & $\epsilon_{r}$ & $\mathrm{E}(\mathrm{GPa})$ & $\begin{array}{l}\mathrm{CTE} \\
(\mathrm{ppm} / \mathrm{K})\end{array}$ \\
\hline $\mathrm{Si}$ [23] & - & 190 & 2.33 \\
$\mathrm{SiO}_{2}$ (PECVD TEOS) [24] & 3.9 & 64 & 2.61 \\
$\mathrm{Si}_{3} \mathrm{~N}_{4}$ (LPCVD) [25] & 7 & 261 & $1.7-2.3$ \\
$\mathrm{BCB}^{2}$ 3000 Series (Dow) [26] & $2.65^{\mathrm{a}}$ & $2.7-3$ & 42.3 \\
Parylene N [27] & $2.65^{\mathrm{b}}$ & 2.4 & 69 \\
SU-8 2000 (MicroChem) [28] & $3.2^{\mathrm{c}}$ & 2 & 52 \\
InterVia 8023 (Dow) [29] & $3.2^{\mathrm{d}}$ & 4 & 62 \\
\hline
\end{tabular}

a $1-20 \mathrm{GHz}$.

${ }^{\mathrm{b}} 60 \mathrm{~Hz}-1 \mathrm{MHz}$.

${ }^{c}$ At $10 \mathrm{MHz}$

${ }^{\mathrm{d}}$ At $1 \mathrm{GHz}$.

which results in corrugated sidewalls. By using state-of-theart DRIE equipment, these effects can be minimized [7] and adopted to the demands of subsequent insulation, barrier and seed-layer deposition steps.

Via insulator. Chemical vapor deposition (CVD) is a well-established CMOS-compatible process with moderate temperature requirements $[1,2,11,9]$ and is therefore the most commonly used method for a direct deposition of silicon dioxide or silicon nitride on via sidewalls. Organic dielectrics $[16,17]$ including benzocyclobutene (BCB) [13, 15, 18, 19], epoxy-based polymers [13, 12], silicone [13] or Parylene [11] are used as well. Polymers, especially low-k types with a lower relative permittivity compared to silicon dioxide, are very attractive for the realization of TSVs with improved electrical characteristics in terms of lower capacitive parasitics $[20,12,15]$. The relative permittivity of selected via insulation materials are listed in table 1 . Furthermore, polymers can act as a buffer for thermo-mechanical stress that is caused by coefficient of thermal expansion (CTE) mismatches between the via metallization and the silicon bulk material $[17,21$, $20,22]$. As shown in table 1 , the Young modulus of these polymers is typically two orders of magnitude lower as compared to silicon dioxide and silicon nitride.

Via conductor. The formation of a low-resistivity via conductor is the most critical and often most costly part of the via fabrication. The two basic via designs are either based on solid or lined metallizations for the vertical conductor. Established processes are electrodeposition of copper [8, 9, 11-13, 22], CVD of tungsten [2, 30], CVD of polysilicon $[2,31]$ and the use of low-resistivity bulk silicon [10]. In particular, electrodeposition of copper, being a very well established semiconductor process, is used by many research groups and implemented in most commercialized devices containing TSVs. Electrodeposition of copper benefits from widely available tool vendor support and process maturity as well as being amenable to deposition at near to room temperature, but suffers due to its complexity in terms of process controllability, reliability and throughput [4]. In particular, it is challenging to implement high aspect ratio TSVs with void-free conductive metal cores [4, 9, 32]. Alternative approaches to plating processes have therefore (a)

$(c)$

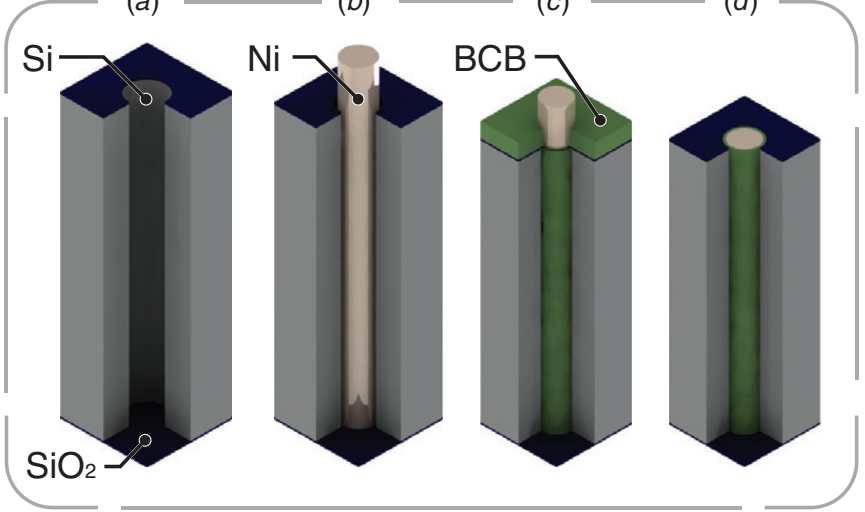

Figure 1. Via formation concept. (a) The via hole is formed by DRIE, stopping on a silicon dioxide layer. (b) A conductive, ferromagnetic nickel core is placed in the via hole by magnetic assembly. (c) The remaining hollow space in the via cavity is filled with the thermosetting polymer BCB. $(d)$ A grinding and polishing step removes excess polymer and nickel from the frontside.

Table 2. Electrical resistivity $\rho$ and mechanical properties (Young's modulus $E$ and coefficient of thermal expansion (CTE)) of common TSV metallizations and ferromagnetic elements. Silicon serves as reference in the first row.

\begin{tabular}{llll}
\hline Material & \multicolumn{1}{c}{$\rho(\Omega \mathrm{m})$} & $E(\mathrm{GPa})$ & $\begin{array}{l}\mathrm{CTE} \\
(\mathrm{ppm} \mathrm{K})^{-1}\end{array}$ \\
\hline $\mathrm{Si}[23]$ & - & 190 & 2.33 \\
$\mathrm{Cu}[39]$ & $1.7 \times 10^{-8}$ & 110 & 16.4 \\
$\mathrm{Tu}[39]$ & $5.65 \times 10^{-8}$ & 400 & 4.4 \\
$\mathrm{Au}[39]$ & $2.2 \times 10^{-8}$ & 77.2 & 14.4 \\
$\mathrm{Ni}[39]$ & $6.4 \times 10^{-8}$ & 207 & 13.1 \\
$\mathrm{Co}[39]$ & $6.24 \times 10^{-8}$ & 211 & 12.5 \\
$\mathrm{Fe}[39]$ & $8.9 \times 10^{-8}$ & 200 & 12.2 \\
\hline
\end{tabular}

been investigated, such as the via filling with conductive metal pastes $[1,33,34]$, solder $[35,36]$ as well as the use of wirebonded metal cores [37, 38, 15].

As shown in table 2, the electrical resistivity of ferromagnetic nickel is similar to tungsten, but approximately three to four times higher as compared to gold and copper. The CTE of nickel is approximately $20 \%$ lower as compared to copper. Volume-manufactured nickel wires with diameters down to $10 \mu \mathrm{m}$ are commercially available and are typically used for chemically resistant woven filter cloth, screen printing masks and recently also for wire bonded interconnections in high-temperature packaging of $\mathrm{SiC}$ electronics [40].

In this work, we present the automated magnetic assembly of solid conductive via cores into TSV holes with very high aspect ratios. Magnetism as a non-contact force enables a controlled manipulation of ferromagnetic features over long distances and is insensitive to the surrounding medium and independent of details of the surface chemistry. Magnetic fields can have high-energy densities and can influence feature sizes from macro- to nano-scale. These advantageous characteristics are very attractive and have been reported in various assembly approaches [41]. The presented concept for the TSV metallization and insulation process enables high aspect ratio vias with an inherently void-free metal core.

As depicted in figure 1 , the filling of the via with a conductive material is not realized by a deposition of a 
metal but by an instant filling technique that magnetically assembles pre-formed conductive via cores into the via holes. The via insulator is a polymer that acts both as low-k electrical insulator and buffer against thermo-mechanically induced stresses. As shown in figures $1(b)$ and $(c)$, the order of the metallization and insulation process step is reversed as compared to most conventional TSV fabrication scenarios where the metallization is gradually grown on barrier, insulation and seed layers. The proposed approach therefore does not require any additional high aspect ratio lithography and patterning of the insulation polymer. More importantly, this insulation technique is insensitive to the topography of the via sidewall (i.e. scallops). The proposed fabrication method enables a cost-effective fabrication of high aspect ratio TSVs especially for low- to medium- $I / O$ density applications such as interposers and MEMS. A proof of concept of the fabrication of TSVs with an aspect ratio of 8 by manual magnetic assembly has been shown by the authors earlier [18, 19]. This method has also been adopted for the assembly of SMD capacitors into through-silicon holes [42].

\section{TSV fabrication by automated magnetic assembly}

\subsection{Robotic assembly setup}

An automated assembly process that utilizes the ferromagnetic properties of nickel has been developed for the placement of nickel cores into via holes. In this process, an excess amount of nickel wires are randomly placed on the frontside of a wafer and assembled into etched via holes. By applying a magnetic field, induced by a permanent magnet from the backside of the wafer, the nickel wires align themselves along the field lines and erect themselves perpendicular to the wafer surface, as shown in figure 2. Because of the magnetic force the wires always will remain above the magnet. This effect allows the nickel wires to be steered around on the wafer surface by simply moving the permanent magnet laterally underneath the wafer. The assembly of the nickel cores is achieved by magnetically moving the wires over the via holes. The upright

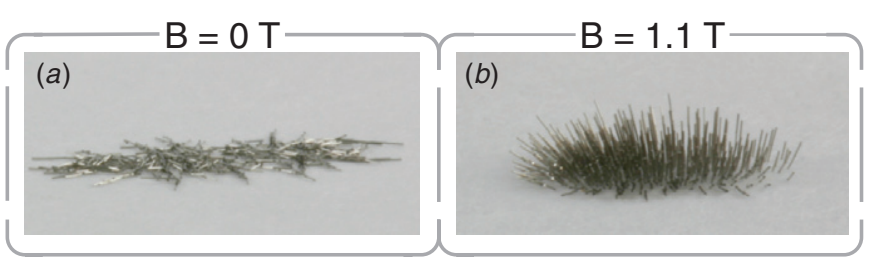

Figure 2. Behaviour of nickel wires in a magnetic field. (a) About 300 straight nickel wires ( $35 \mu \mathrm{m}$ diameter, $350 \mu \mathrm{m}$ length) without an applied field. (b) A magnetic field of $1.1 \mathrm{~T}$ is generated by a cylindrical permanent magnet. It aligns the nickel wires along the field lines perpendicular to the ground plane.

position of the wires allows them to be pulled into the holes by the magnet.

For this magnetic assembly process a robotic setup has been devised. It is based on a handler robot (IWH-series) for $200 \mathrm{~mm}$ wafers from Isel Germany AG. Figure 3 shows a schematic depiction of the robot arm that has been modified in order to mount a cubic permanent magnet with an edge length of $5 \mathrm{~mm}$. This tool enables a programmable movement of the magnet with three degrees of freedom at a precision of $30 \mu \mathrm{m}$. That way different movement patterns were implemented and adopted to varying layouts of via holes. Also depicted in figure 3 is a camera that is mounted directly above the magnet and faces the frontside of the substrate. It is used to optically inspect the substrate surface before and after the assembly and to monitor the assembly process. Furthermore, the tool still retains its capability to handle wafers with the wafer gripper that is a part of the robot arm. This robot arm has a movement range of $\pm 240^{\circ}$ around its axis, $\pm 366 \mathrm{~mm}$ in the radial direction and $323 \mathrm{~mm}$ in the vertical direction. The maximum speeds are $360^{\circ} \mathrm{s}^{-1}, 1000 \mathrm{~mm} \mathrm{~s}^{-1}$ radial and $450 \mathrm{~mm} \mathrm{~s}^{-1}$ vertical. By using a wafer handler robot for the magnetic assembly, an automated cassette-to-cassette process can be implemented, i.e. picking a wafer from a input/output cassette, placing it on a dedicated assembly stage, performing the magnetic assembly and placing the wafer back into the cassette.
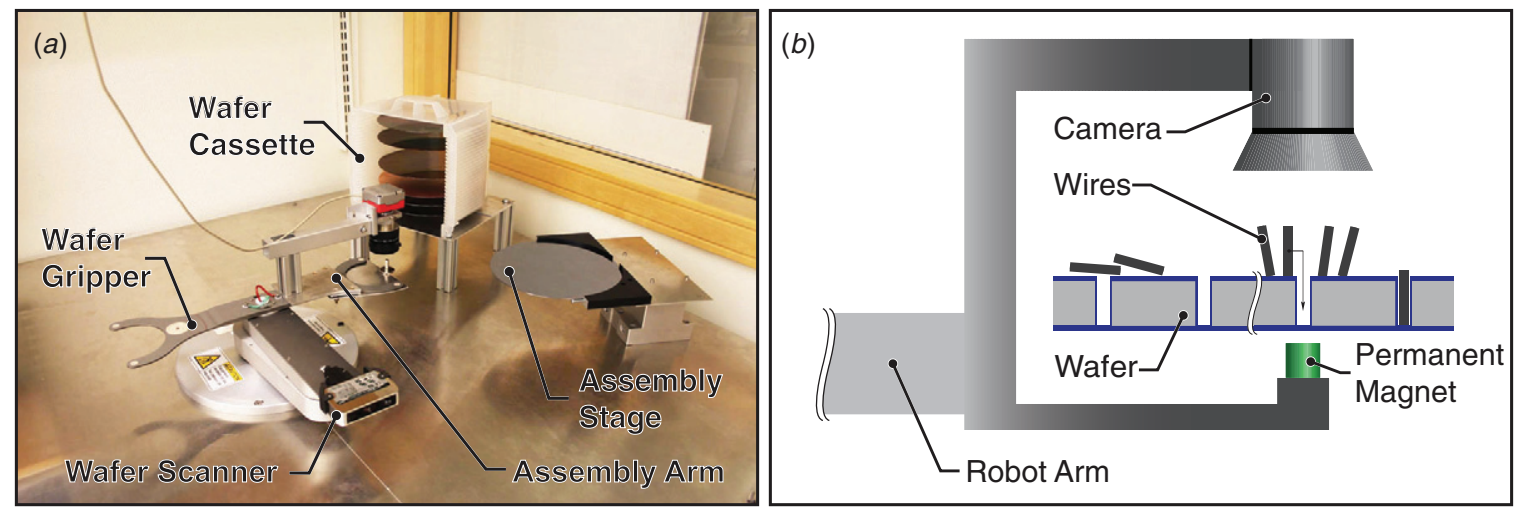

Figure 3. (a) Assembly setup with the wafer gripper and assembly part on the robot arm in the centre of the table, the wafer cassette station and the assembly stage to its right. The assembly process consists of four steps: (1) scanning the cassette for wafers, (2) picking the chosen wafer and placing it on the assembly stage, (3) positioning the assembly arm, placing the magnetic via cores manually on the substrate and carrying out the automated magnetic assembly, (4) putting the wafer back into the cassette. (b) Schematic drawing showing the custom-built assembly arm that consists of a permanent magnet mounted on an aluminium sheet and a camera above the magnet. 


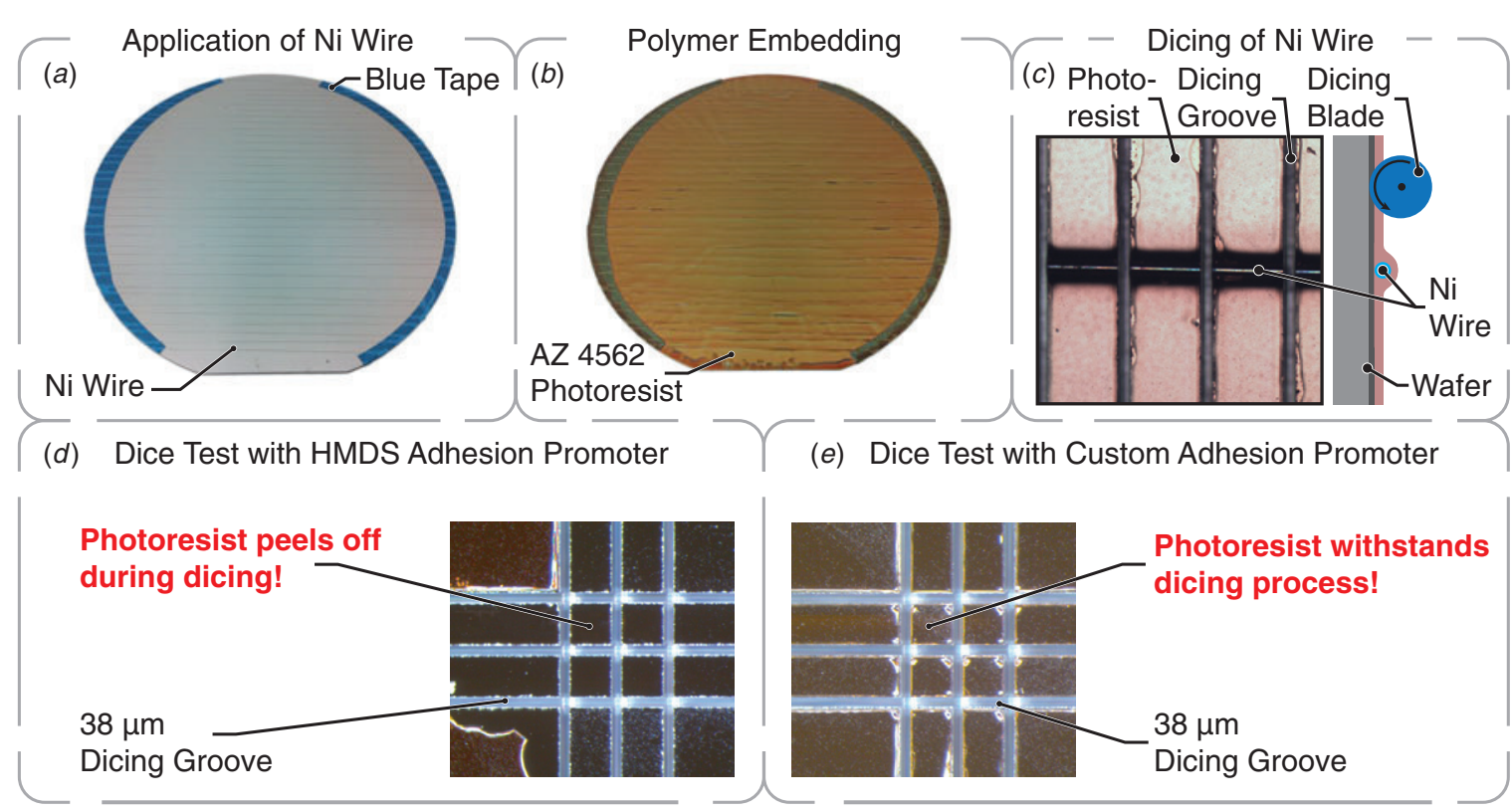

Figure 4. Nickel wire preparation. (a) The nickel wires are manually placed on a dummy wafer and fixated at the outer perimeter of the carrier with the help of blue tape. (b) The wires are then embedded in a matrix of photoresist and subsequently diced. $(c)$ The microscopic image of a diced nickel wire with a diameter of $35 \mu \mathrm{m}$. $(d)$ The microscopic image of a dicing test with a pitch of $150 \mu \mathrm{m}$ with photoresist and standard HMDS adhesion promoter. The photoresist peels off during dicing. The adhesion showed to be insufficient for dicing pitches below $500 \mu \mathrm{m}$. (e) The microscopic image of a dicing test with a pitch of $150 \mu \mathrm{m}$ with photoresist and custom adhesion promoter. The resist fully adheres to the substrate.

\subsection{Nickel wire preparation}

In order to cut a nickel wire into rods of a defined length, a cutting process was developed. For the experiments, Ni-270 wire with a purity of $99.97 \%$ with two different diameters was used, 15 and $35 \mu \mathrm{m}$. As depicted in figure 4(a), several wires are placed in parallel on a silicon carrier wafer and subsequently embedded in a polymer matrix (figure $4(b)$ ) in order to safely fixate the wires on the carrier wafer and to minimize the deformation and burr creation during the cutting process. The wires are then cut with the help of a wafer dicing tool (figure $4(c)$ ) and finally released by dissolving the polymer in a solvent.

A good adhesion of the photoresist to the silicon substrate is essential in order to ensure a proper fixation of the wire rods during the dicing process. Figure $4(d)$ depicts that the photoresist tends to peel off with decreasing dicing pitch (i.e. nickel rod length) on substrates that are treated with standard hexamethyldisilazane (HMDS) as an adhesion promoter. In order to increase the yield of the wire cutting process, the bond strength between the carrier substrate and the novolacbased photoresist was increased by a custom-made adhesion promoter. The silicon carrier substrate was immersed in a solution of 5\% 3-(triethoxysilyl)propylsuccinicanhydride and $95 \%$ Toluene for $10 \mathrm{~min}$. The wafer was then rinsed with Toluene, blow-dried and finally baked in a oven at a temperature of $105^{\circ} \mathrm{C}$ for $10 \mathrm{~min}$. The applied silane reacts with the wafer surface via a silanization reaction, forming a very thin, covalently bonded organic layer. Upon completion of the silanization step, a very dense concentration of anhydride groups is exposed on the surface. Anhydride groups readily react with the hydroxyl groups present in the uncured novolac photoresist, that is applied later on. After the aforementioned treatment, the wires were manually placed on a dummy wafer and fixated with blue tape at the outer perimeter of a carrier, as shown in figure $4(a)$. A layer of $\mathrm{AZ}^{\circledR} 4562$ photoresist was then spin-coated on the carrier wafer at $1000 \mathrm{rpm}$ for $30 \mathrm{~s}$. The photoresist was soft baked on a hotplate at a temperature of $50{ }^{\circ} \mathrm{C}$ for $3 \mathrm{~min}$. A low soft-baking temperature was chosen in order to retain sufficient elastic properties of the polymer. As depicted in figure $4(c)$, the nickel wires were fully embedded in the photoresist matrix. A DAD 320 (DISCO Corporation, Japan) wafer dicing tool that was equipped with a $38 \mu \mathrm{m}$ wide dicing blade was used to cut the wires. The dicing feed speed was $10 \mathrm{~mm} \mathrm{~s}^{-1}$. The accurate alignment of the dicing tool allowed for perfectly perpendicular cuts and the length of the rods could be precisely controlled by the dicing tool. Figure 4(e) shows that the resist fully adheres to the silicon surface and does not peel off for dicing pitches of $150 \mu \mathrm{m}$. That way a yield of $100 \%$ for the cutting process could be achieved. Subsequently, the nickel rods could easily be released by dissolving the resist layer with acetone. This process allows a very precise cutting of many wires in parallel and can produce several thousands of nickel rods per run. Laser cutting or automated wire cutting tools may serve as an alternative method especially for a high-volume production of nickel rods.

\subsection{TSV fabrication}

The fabrication process for the TSVs is illustrated in figure 5 and is based on double-side polished $100 \mathrm{~mm} \mathrm{Si}$ wafers with a $2 \mu \mathrm{m}$ thick silicon dioxide layer on both sides, which was created by thermal wet oxidization at $1100{ }^{\circ} \mathrm{C}$. The oxide acts 


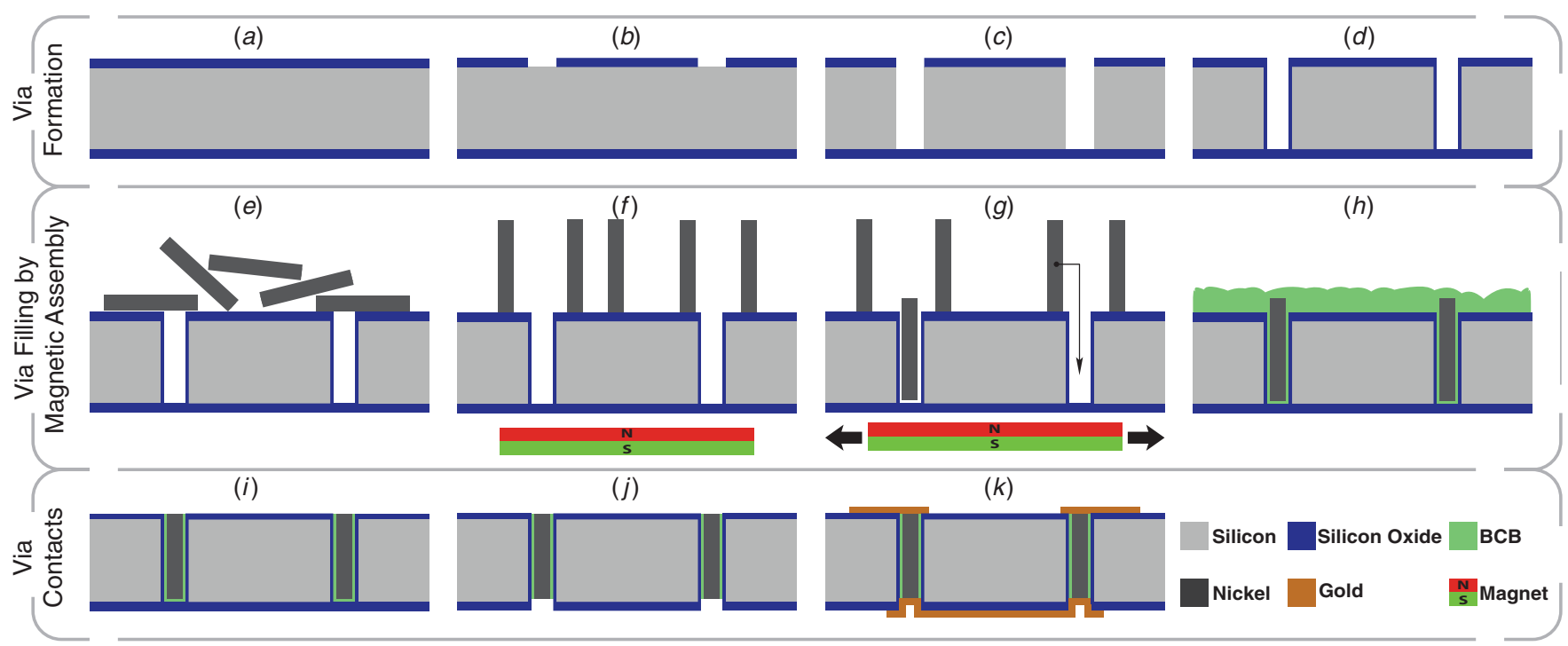

Figure 5. The TSV fabrication scheme can be divided into three main steps. First is the formation of the via hole by DRIE etching, second is the magnetic assembly of the conductive TSV core and third is the filling with the dielectric.

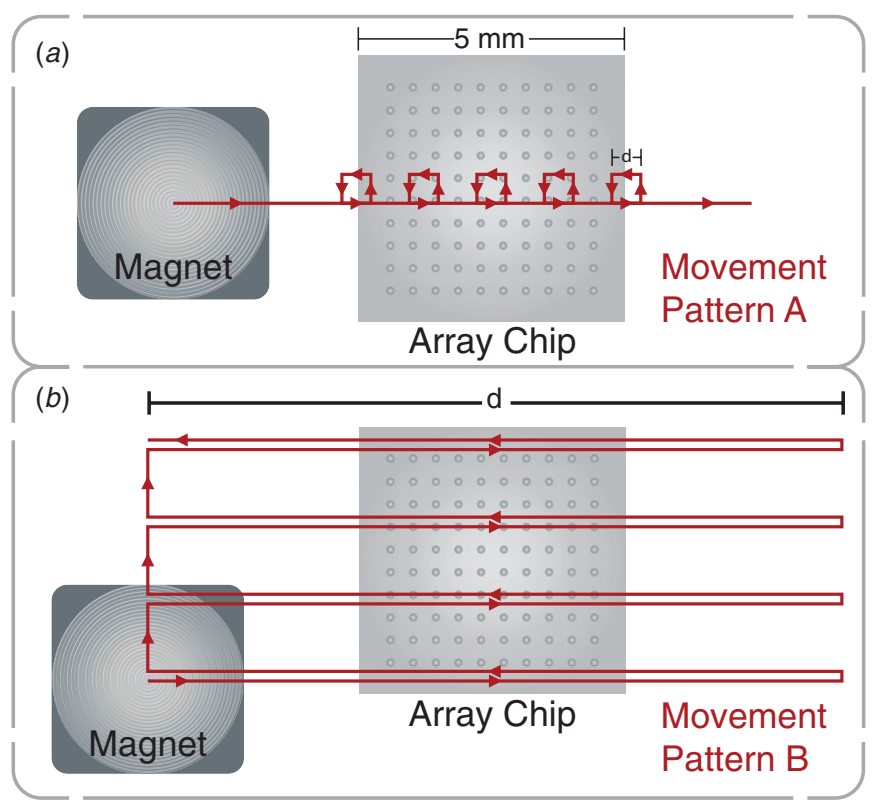

Figure 6. Movement patterns of the assembly robot. (a) Small advancing rectangles with an edge length of $d=0.76 \mathrm{~mm}$. (b) Long linear sweep with a length of approximately $d=1.5 \mathrm{~cm}$. The sweeping is based on a radial movement of the robotic arm. In both illustrations the sweeps are approximated to straight linear movements due to the large radius of the radial movement (approximately $0.75 \mathrm{~m}$ ) and the comparable short sweep length $d$.

both as a hard mask for the DRIE step and as an electrical insulator for the metal lines, which will finally connect the via on the frontside and backside of the substrate. A standard lithography on the frontside of the substrate defines the circular openings for the vias. The silicon dioxide is dry-etched by RIE, as illustrated in figure 5(b). As depicted in figure 5(c), a Bosch DRIE process creates via holes with straight side walls. The DRIE stops at the silicon dioxide on the bottom of the cavity. A subsequent high temperature treatment at $1100{ }^{\circ} \mathrm{C}$ in a furnace is used to remove polymer residuals from the
DRIE passivation cycles by pyrolization. In the same furnace, a thermal oxidation at $1100{ }^{\circ} \mathrm{C}$ creates a $0.5 \mu \mathrm{m}$ thick silicon dioxide layer, as shown in figure $5(d)$. The silicon dioxide layer ensures an electrical insulation of the via sidewalls and creates a hydrophilic surface on the via sidewall, which is of importance for the insulation step that is carried out later on.

The pre-fabricated nickel cores are then distributed on the frontside of the target wafer. By utilizing the robotic assembly tool, the permanent magnet on the assembly arm can be moved into close proximity of the backside of the wafer, as indicated in figure $5(f)$. The nickel wires that are manually placed on the wafer surface are drawn to the location of the magnet and erect themselves perpendicular to the substrate surface. With programmed patterns for the magnet movement, all via holes can be filled in an automated process. The results of the performed assembly experiments, including the movement patterns and the yield of the filling process, are presented in section 4.

The via cavities are subsequently filled with the thermosetting polymer BCB CYCLOTENE ${ }^{\circledR} \quad 3022-46$ (figure $5(g)$ ), which is known to be suited for a void-free filling of high aspect ratio features [43]. In order to reduce the viscosity of the polymer, the substrate is placed on a hotplate with a temperature of $60{ }^{\circ} \mathrm{C}$ before the polymer is manually applied to the wafer surface using a syringe. As the polymer is not spin-coated, the resulting polymer layer has a non-uniform thickness on the order of 100-150 $\mu \mathrm{m}$. The subsequent hard-curing of the $\mathrm{BCB}$ is performed on a hotplate in a vacuum chamber using the temperature profile according to the manufacturer's standard process procedures [26]. The entire curing procedure was performed in a vacuum environment at $0.02 \mathrm{mbar}$ in order to prevent any void formation in the polymer. A subsequent grinding and polishing step removes excess nickel and BCB from the surface of the substrate, as shown in figure 5(i). A lithography and RIE of the silicon dioxide and $\mathrm{BCB}$ residues opens the contact area of the via on the backside of the wafer, as illustrated in 
figure $5(j)$. Two consecutive TiW/Au depositions $(50 / 1000 \mathrm{~nm})$ on both sides of the wafer interconnects the nickel cores of the vias. A lithography, wet Au etch and dry TiW etch (figure $5(k)$ ) are made to define the Kelvin test structures.

\section{Experimental results}

The performance of the robotic assembly setup in terms of assembly speed was evaluated and an optical inspection and an electrical characterization of the fabricated TSVs were performed.

\subsection{Automated magnetic assembly}

A series of assembly experiments with different movement parameters was conducted. As shown in figure 6, the magnetic assembly process was performed on array structures of via holes with an excessive amount of nickel wires of approximately 2500-3000. The wires in these experiments had a diameter of $35 \mu \mathrm{m}$ and a length of $360 \mu \mathrm{m}$. The via holes had a diameter of $42 \mu \mathrm{m}$ and were fabricated in arrays of $10 \times$ 10 vias with a pitch of $350 \mu \mathrm{m}$ on a substrate with a thickness of $350 \mu \mathrm{m}$. With respect to the array size of $3.5 \mathrm{~mm}$ and the size of the cubic permanent magnet with an edge length of $5 \mathrm{~mm}$, two different movement patterns were programmed, as schematically illustrated in figure 6 . The pattern in figure $6(a)$ has a very short sweep length $d$ of $0.76 \mathrm{~mm}$, whereas the pattern in figure $6(b)$ sweeps over the array with a sweep length $d$ of $1.5 \mathrm{~cm}$. Furthermore, the experiments have been conducted at two assembly motion speeds, a fast motion at $120{ }^{\circ} \mathrm{s}^{-1}$ and a slow motion at $4{ }^{\circ} \mathrm{s}^{-1}$. The vertical distance of the magnet to the backside of the wafer was approximately $125 \mu \mathrm{m}$ in all experiments.

As figure 7 shows, the filling rate, i.e. the number of filled via holes per second, is dependent on the speed and the sweep length $d$ of the assembly motion. Larger sweep lengths result in a faster filling process. The lateral force on the wires only

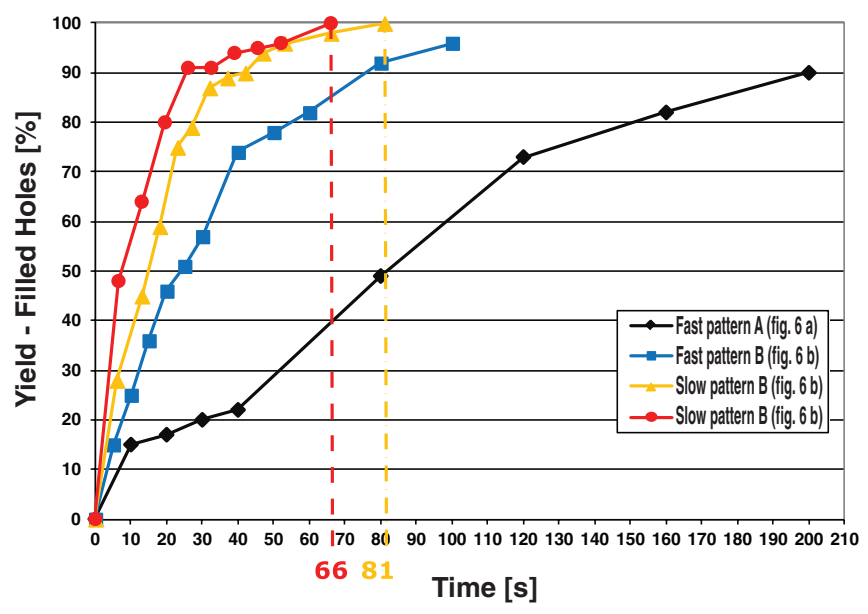

Figure 7. Filling rates for the assembly of via arrays $(10 \times 10$ holes). Two different movement patterns and two different speeds were tested. The slow patterns exhibited a yield of $100 \%$ and the highest filling rate.

occurs due to a gradient in the magnetic field, which is more prominent near the edge of the cubic magnet. For small sweep lengths, this implies that the wires located above the centre of the magnet move very little or not at all, which explains the poor filling rate from the movement pattern shown in figure $6(a)$. Also shown in figure 7 , the assembly process improves with decreasing speed of the assembly motion. A fast assembly motion causes the wires to tilt from their perpendicular position while being dragged along the substrate surface which makes it more difficult to pull them into the via holes. Slower motion speeds reduce the wire tilt and therefore increase the filling rate. As shown in figure 7, a filling yield of $100 \%$ within 66 to $81 \mathrm{~s}$ could be demonstrated by using the movement pattern indicated in figure $6(b)$ and a slow motion speed of $4{ }^{\circ} \mathrm{s}^{-1}$.

As shown in figure 8 , assembled wires that are protruding the substrate surface act both as a mechanical and magnetic obstruction for the excess wires during the assembly process.

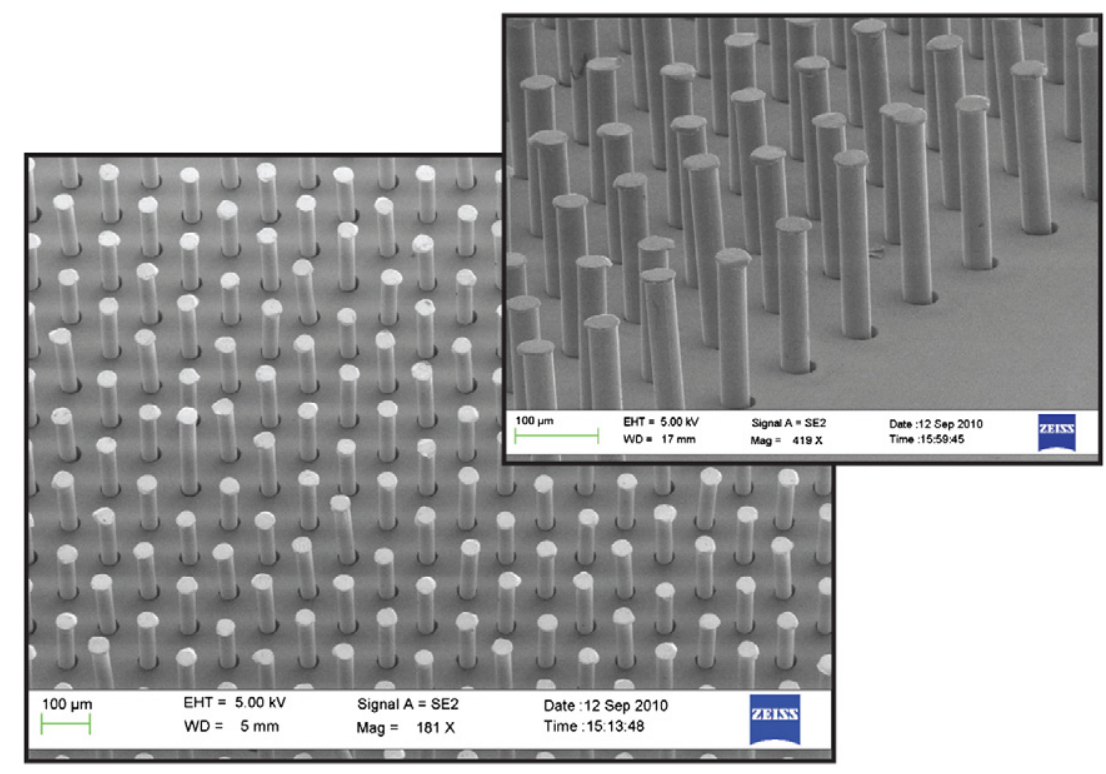

Figure 8. SEM image of a $30 \times 30$ array with a pitch of $120 \mu \mathrm{m}$ of nickel wires placed in the via hole prior to the filling of BCB. The minimum via hole diameter for a $35 \mu \mathrm{m}$ wire was determined to be $40 \mu \mathrm{m}$. 


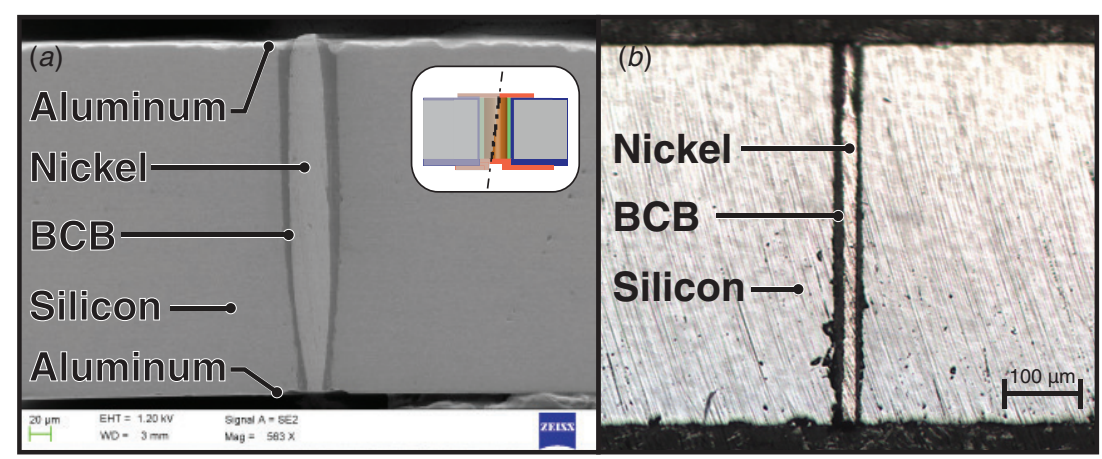

Figure 9. (a) SEM image of a polished cut through a TSV with an aspect ratio of 8 . Note: as indicated in the drawing, the sample was tilted during the grinding process of the cross section, which leads to the apparent view of a non-constant via diameter, from [18]. (b) Optical microscope image of a polished cut through a TSV with an aspect ratio of 31 for the metal core and an overall aspect ratio of 24.

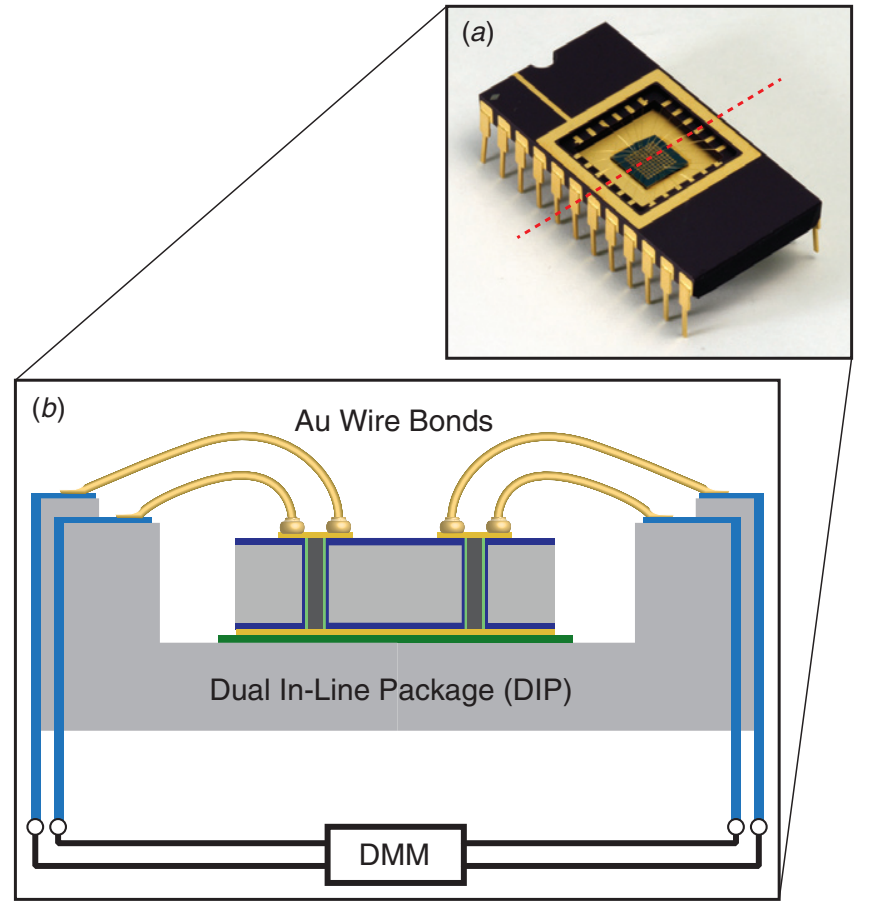

Figure 10. (a) Test chip with a $10 \times 10$ TSV array that has been mounted in the cavity of a 24-pin dual in-line package (DIP).

(b) Illustration of the contacting scheme for the four-wire resistance measurement of a chain of two TSVs.

These two effects have a negative impact on the filling rate. In order to overcome the mechanical obstruction, the wires can be cut to a length that is the same or shorter as the depth of the via holes. Due to the magnetization of the nickel during the assembly process, the excess wires can stick to the ends of assembled wires and cluster even if the assembly-magnet moves on. It is possible to demagnetize and thereby eliminate the clustering of the ferromagnetic nickel rods by reversing the magnetic field by either flipping the permanent magnet or using an alternating magnetic field that is induced by a electromagnet. By flipping the permanent magnet it was even possible to fill a via array with a very dense pitch of $120 \mu \mathrm{m}$ with wires that were considerably protruding the surface by approximately $150 \mu \mathrm{m}$, as shown in figure 8 .

Potentially, the assembly process can be improved and further accelerated by the implementation of a direct optical inspection feedback. The camera, depicted in figure 3, that is mounted on the assembly robot has only been used for monitoring the assembly process in our experiments. By using an automated pattern recognition, the software can be extended to be able to identify filled and empty holes. This information can then be used to implement a feedback loop for the assembly movement. Thus, an adaptive optimization of the assembly movement during operation can be obtained. Moreover, this addition would provide the assembly process with an inherent quality control functionality that detects unfilled via holes.

\subsection{Cross-section inspection of filled TSVs}

In order to evaluate the filling with dielectric and nickel, several polished cuts through magnetically assembled TSVs have been prepared and inspected by optical microscopy and scanning electron microscopy (SEM). As shown in figures $9(a)$ and $(b)$, the filling with BCB was successfully conducted without any visible air-voids or defects after the complete hard curing procedure. Also, due to the use of wire as a base material, the nickel core is inherently void-free. There are no indications for delamination of the $\mathrm{BCB}$ at the via side walls or the via core, which might cause mechanical or electrical failures of the vias. Figure $9(a)$ shows the cross-section of a magnetically assembled TSV with an aspect ratio of 8 that consists of a nickel core with diameter of $35 \mu \mathrm{m}$, a length of $325 \mu \mathrm{m}$ and a via hole diameter of $40 \mu \mathrm{m}$. As depicted in figure $9(b)$, a similar inspection was performed with a magnetically assembled TSV with an aspect ratio of approximately 24 . This TSV consists of a nickel core with diameter of $15 \mu \mathrm{m}$ and a length of $470 \mu \mathrm{m}$ that was assembled in a via hole with a diameter of $20 \mu \mathrm{m}$.

\subsection{Electrical characterization}

The electrical resistance of magnetically assembled nickel TSVs with a diameter of $35 \mu \mathrm{m}$ and a length of $250 \mu \mathrm{m}$ has been evaluated. A chip with a $10 \times 10$ array of TSVs with a pitch of $350 \mu \mathrm{m}$ was mounted in a ceramic dual inline package with an open cavity (figure 10(a)). As illustrated in figure $10(b)$, each via was contacted on the frontside with two wire bonds that have been placed with an automatic wire bonder model ESEC $3100+$. On the backside, the vias are 
electrically connected by a blank metallization. A four-wire measurement was performed on 25 pairs of TSVs (i.e. 50 TSVs) using a HP 34401A digital multimeter. The measured total resistance is in average $83 \mathrm{~m} \Omega$ for two TSVs, including their contact metallization on the frontside and backside of the chip. This is in good agreement with the theoretical resistance of one pair of $\mathrm{Ni}$ TSVs of the given dimensions, which is approximately $40 \mathrm{~m} \Omega$, excluding any contact metallization.

\section{Conclusions}

The fabrication of TSVs with aspect ratios of up to 24 based on an automated magnetic assembly process has been demonstrated. Smaller TSV diameters and higher aspect ratios are feasible but limited by the smallest commercially available diameter of ferromagnetic wires, which is to our knowledge currently $10 \mu \mathrm{m}$ for nickel. The novel automated magnetic assembly process enables a high-speed filling of TSV holes with high aspect ratio via cores for low- to mediumI/O density applications such as interposers and MEMS. The presented concept addresses main fabrication objectives relevant for state-of-the-art, low-resistance metal TSVs such as reliable fabrication of high aspect ratio vias, void-free solid metallizations, sufficient thermo-mechanical stability and reduction of fabrication costs.

\section{Acknowledgments}

This work has been funded in part by the European Research Council (ERC) through the Starting grant (no 277879). The authors also would like to thank Nora Heinig for her collaboration and technical support.

\section{References}

[1] Motoyoshi M 2009 Through-silicon via (TSV) Proc. IEEE 97 43-8

[2] Koyanagi M, Fukushima T and Tanaka T 2009 High-density through silicon vias for 3D LSIs Proc. IEEE 97 49-59

[3] Weerasekera R, Pamunuwa D, Zheng L-R and Tenhunen H 2009 Two-dimensional and three-dimensional integration of heterogeneous electronic systems under cost, performance and technological constraints IEEE Trans. Comput.-Aided Des. Integr. Circuits Syst. 28 1237-50

[4] Garrou P, Bower C and Ramm P 2008 Handbook of 3D Integration Technology and Application of $3 D$ Integration Circuits (New York: Wiley)

[5] Lau J, Lee R, Yuen M and Chan P 2010 3D LED and IC wafer level packaging Microelectron. Int. 27 98-105

[6] Lapisa M, Stemme G and Niklaus F 2011 Wafer-level heterogeneous integration for MOEMS, MEMS and NEMS IEEE J. Sel. Top. Quantum Electron. 17 629-44

[7] Tezcan D, Munck K De, Pham N, Luhn O, Aarts A, De Moor P, Baert K and Van Hoof C 2006 Development of vertical and tapered via etch for $3 \mathrm{~d}$ through wafer interconnect technology EPTC'06: Electronics Packaging Technology Conf. (8 Dec.) pp 22-28

[8] Nilsson P, Ljunggren A, Thorslund R, Hagstrom M and Lindskog V 2009 Novel through-silicon via technique for $2 \mathrm{~d} / 3 \mathrm{~d}$ sip and interposer in low-resistance applications ECTC'09: 59th Electronic Components and Technology Conf. pp 1796-1801
[9] Wolf M, Dretschkow T, Wunderle B, Jurgensen N, Engelmann G, Ehrmann O, Uhlig A, Michel B and Reichl H 2008 High aspect ratio TSV copper filling with different seed layers ECTC'08: 58th Electronic Components and Technology Conf. pp 563-70

[10] Rimskog M 2007 Through wafer via technology for MEMS and 3d integration IEMT'07: 32nd IEEE/CPMT Electronic Manufacturing Technology Symp. pp 286-9

[11] Tezcan D, Pham N, Majeed B, Moor P De, Ruythooren W and Baert K 2007 Sloped through wafer vias for 3d wafer level packaging ECTC'07: Proc. 57th Electronic Components and Technology Conf. (29 May-1 June) pp 643-7

[12] Ho S W, Yoon S W, Zhou Q, Pasad K, Kripesh V and Lau J 2008 High RF performance TSV silicon carrier for high frequency application ECTC'08: 58th Electronic Components and Technology Conf. pp 1946-52

[13] Tezcan D, Duval F, Philipsen H, Luhn O, Soussan P and Swinnen B 2009 Scalable through silicon via with polymer deep trench isolation for $3 \mathrm{~d}$ wafer level packaging ECTC'09: 59th Electronic Components and Technology Conf. pp 1159-64

[14] Tang C W, Young H T and Li K M 2012 Innovative through-silicon-via formation approach for wafer-level packaging applications J. Micromech. Microeng. 22045019

[15] Fischer A, Grange M, Roxhed N, Weerasekera R, Pamunuwa D, Stemme G and Niklaus F 2011 Wire-bonded through-silicon vias with low capacitive substrate coupling J. Micromech. Microeng. 21085035

[16] Sundaram V, Chen Q, Suzuki Y, Kumar G, Liu F and Tummala R 2012 Low-cost and low-loss 3d silicon interposer for high bandwidth logic-to-memory interconnections without TSV in the logic IC ECTC'12: 62th Electronic Components and Technology Conf. pp 292-7

[17] Wang M-J, Hung C-Y, Kao C-L, Lee P-N, Chen C-H, Hung C-P and Tong H-M 2012 TSV technology for 2.5d IC solution ECTC'12: 62th Electronic Components and Technology Conf. pp 284-8

[18] Fischer A, Roxhed N, Haraldsson T, Heinig N, Stemme G and Niklaus F 2011 Fabrication of high aspect ratio through silicon vias (TSVs) by magnetic assembly of nickel wires MEMS'11: IEEE 24th Int. Conf. on Micro Electro Mechanical Systems pp 37-40

[19] Fischer A C, Bleiker S J, Somjit N, Roxhed N, Haraldsson T, Stemme G and Niklaus F 2012 High aspect ratio TSVs fabricated by magnetic self-assembly of gold-coated nickel wires ECTC'12: 62nd Electronic Components and Technology Conf. pp 541-7

[20] Civale Y, Tezcan D, Philipsen H, Duval F, Jaenen P, Travaly Y, Soussan P, Swinnen B and Beyne E 2011 3-d wafer-level packaging die stacking using spin-on-dielectric polymer liner through-silicon vias IEEE Trans. Compon. Packag. Manuf. Technol. $1833-40$

[21] Liu X, Chen Q, Dixit P, Chatterjee R, Tummala R and Sitaraman S 2009 Failure mechanisms and optimum design for electroplated copper through-silicon vias (TSV) ECTC'09: 59th Electronic Components and Technology Conf. pp 624-9

[22] Lu K, Zhang X, Ryu S-K, Im J, Huang R and Ho P 2009 Thermo-mechanical reliability of 3-d ICS containing through silicon vias ECTC'09: 59th Electronic Components and Technology Conf. pp 630-4

[23] Petersen K E 1982 Silicon as a mechanical material Proc. IEEE 70 420-57

[24] Zhao J-H, Ryan T, Ho P S, McKerrow A J and Shih W-Y 1999 Measurement of elastic modulus, poisson ratio and coefficient of thermal expansion of on-wafer submicron films J. Appl. Phys. 85 6421-4 
[25] Chuang W-H, Luger T, Fettig R and Ghodssi R 2004 Mechanical property characterization of LPCVD silicon nitride thin films at cryogenic temperatures Microelectromech. Syst. J. 13 870-9

[26] The Dow Chemical Company 2008 Processing Procedures for Cyclotene 3000 Series Resins http://www.dow.com/ cyclotene/docs/cyclotene_3000_dry_etch.pdf

[27] Parylene Coating Services, Inc. 2011 Properties of Parylene http://www.paryleneinc.com/pdf/PDS_Dimer_ International.pdf

[28] MicroChem 2009 Processing Guidelines for su-8 2000 Permanent Epoxy Negative Photoresist http://www.microchem.com/pdf/SU-82000DataSheet2000_ 5thru2015Ver4.pdf

[29] Rohm and Haas 2009 Intervia Photodielectric 8023 Series http://www.microchem.com/PDFs_Dow/Intervia\% 20Photodielectric\%208023\%20UL-PF08N013R2.pdf

[30] Kikuchi H, Yamada Y, Ali A M, Liang J, Fukushima T, Tanaka T and Koyanagi M 2008 Tungsten through-silicon via technology for three-dimensional LSIs Japan. J. Appl. Phys. 47 2801-6

[31] Dixit P, Vehmas T, Vähänen S, Monnoyer P and Henttinen K 2012 Fabrication and electrical characterization of high aspect ratio poly-silicon filled through-silicon vias J. Micromech. Microeng. 22055021

[32] Gu C, Xu H and Zhang T-Y 2009 Fabrication of high aspect ratio through-wafer copper interconnects by reverse pulse electroplating J. Micromech. Microeng. 19065011

[33] Ham Y-H, Kim D-P, Park K-S, Jeong Y-S, Yun H-J, Baek K-H, Kwon K-H, Lee K and Do L-M 2011 Dual etch processes of via and metal paste filling for through silicon via process Thin Solid Films 519 6727-31

[34] Lee S, Hon R, Zhang S and Wong C 2005 3d stacked flip chip packaging with through silicon vias and copper plating or conductive adhesive filling Proc. 55th Electronic Components and Technology Conf. (31 May-3 June 2005) vol 1 pp 795-801

[35] Ko Y-K, Fujii H T, Sato Y S, Lee C-W and Yoo S 2012 High-speed TSV filling with molten solder Microelectron. Eng. 89 62-4

[36] Gu J, Pike W and Karl W 2009 A novel capillary-effect-based solder pump structure and its potential application for through-wafer interconnection J. Micromech. Microeng. 19074005

[37] Baron J 2010 Stud bumping serves as TSV alternative for BSI image sensor in latest iPhone 4 Yole Development Technical Report 17

[38] Jackson N and Muthuswamy J 2009 Flexible chip-scale package and interconnect for implantable MEMS movable microelectrodes for the brain J. Microelectromech. Syst. 18 396-404

[39] Material Property Database http://www.matweb.com/

[40] Burla R, Chen L, Zorman C and Mehregany M 2009 Development of nickel wire bonding for high-temperature packaging of SiC devices IEEE Trans. Adv. Packag. 32 564-74

[41] Mastrangeli M, Abbasi S, Varel C, Hoof C Van, Celis J and Böhringer K 2009 Self-assembly from milli- to nanoscales: methods and applications $J$. Micromech. Microeng. 19083001

[42] Hoo J, Park K, Varel C, Baskaran R and Böhringer K 2012 Wafer-level high density integration of surface mount technology components in through-silicon trenches MEMS'12: IEEE 24th Int. Conf. on Micro Electro Mechanical Systems pp 373-6

[43] Kotb H M, Isoird K, Morancho F, Théolier L and Do Conto T 2009 Filling of very deep, wide trenches by benzocyclobutene polymer Microsyst. Technol. 15 1395-400 